\title{
A View from the Track: Measuring Spatial Quality of Slow Mobility Routes. Possible Integration of GIS and Machine Learning Based Methods
}

\author{
Alessandro Bianchi \\ Domenico D'Uva \\ Andrea Rolando \\ Alessandro Scandiffio
}

Abstract

The specific aim of this research is to assess the spatial quality of a slow mobility route, integrating two sources of information: the first one based on a quantitative method and the second one focused on a more qualitative oriented one. The first one is a GIS-based analysis which corresponds to a 'looking from above', based on a geographic approach. The second one is based on the 'street level' approach, that is more perceptive, by using Machine Learning techniques based on Google Street View images. The distinction of this work from similar research is related to its focus on the outdoors. The Spatial Quality Index of Slow Routes (SQISR) method allows to compute an index on the base of a set of heterogeneous indicators, by using GIS mapping techniques. The second method is a peculiar application of Image-Recognition technology based on Machine Learning tools, which analyses web images. This methodology produces a set of words, which characterizes the spatial components of the landscape crossed by the route, by arranging the tags into four semantic fields: urban, outdoors, road and car. The applied methodologies give heterogeneous results when valuable elements are far from route. Results correlate in areas where natural elements are present. Further evolution of this research will be based on Machine Learning Image Library customization and improved code-based data acquiring methodology.

\section{Keywords}

GIS, Machine Learning, slow mobility, Spatial Quality Index.

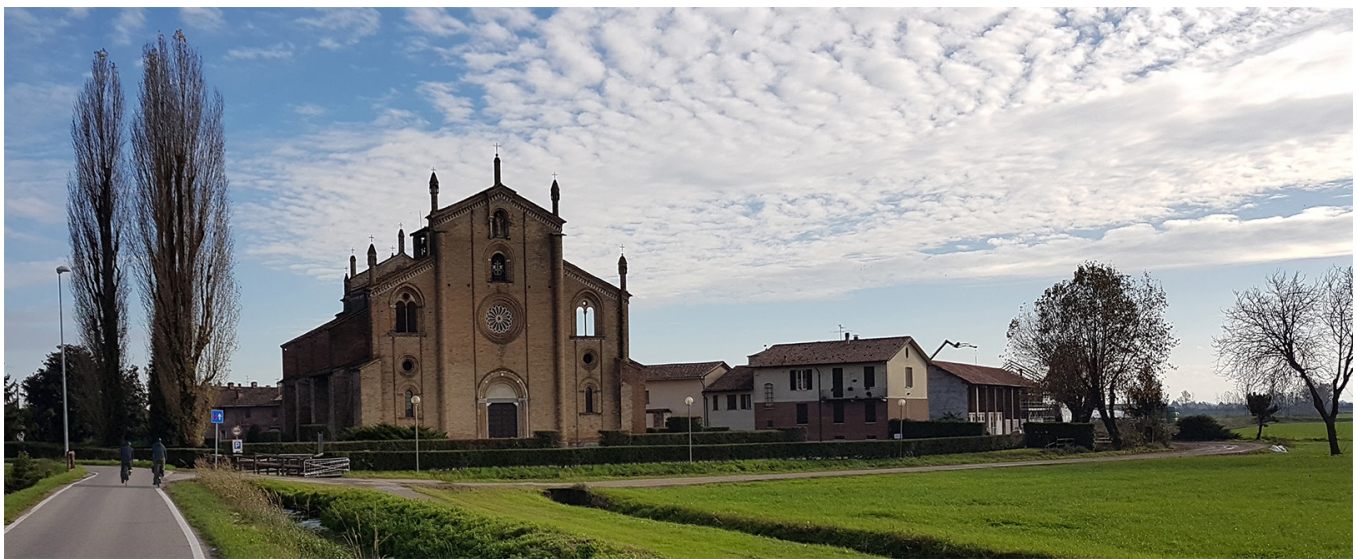




\section{Introduction}

Nowadays, geo-referred informations and digital services enable a throughout knowledge of the features of a territory, that can be useful for insider users (such as inhabitants) and for specific outsider categories (such as tourists). Both populations can therefore produce flows of data that are stored through the internet and that could be easily shared, answering to a specific demand of information [Rolando, Scandiffio 20 I 8]. This paper proposes a study about how to access and use these informations to measure the spatial quality of places.

The work is experimental and tentative, considering that it is important to define an index of quality for several reasons:

- to find common criteria to define and assess spatial quality, considering at the same level both perceptive based approaches (photographs, semantic analysis) and more objective tools (GIS) and also considering possible diverging criteria used by different actors;

- to create a tool able to grade quality according to users' defined parameters, useful for instance to help in choosing a route customized one's needs;

- to help decision makers to concentrate resources on those routes that perform better according to quality performance;

- to encourage confrontation of best practices among local communities, so to improve good design solutions and an overall spatial quality.

A grading of what is quality can be based on objective data, given through a top-down procedure and recognized by most (i.e. a monument, a park) as well as on bottom-up processes, based on personal experience, often influenced by cultural factors (local communities, food, personal interests).

The specific aim of this research is to assess the quality of a slow mobility route, integrating two distinct perceptive approaches, related to different sources of information: the first one based on a more quantitative method and the second one based on a more qualitative oriented one:

- the first is the one that we could define as "looking from above", based on a geographic approach, processing geographic data with GIS analysis and mapping tools and starting from a distant abstract observation point;

- the second is so called "moving through", based on direct experience and, therefore on a more perceptive approach, using machine learning techniques based on images taken from Google Street view, Mapillary or directly from users.

\section{Literature review of related works}

A recent paper about the automated analysis of images' content [Zhang, Zhou, Ratti 2019] proposes a data-driven method to explore the place-informative scenes, by employing deep convolutional neural network to identify and select the features of a place, starting from its image appearance, automatically from a massive dataset of photos. The visual cues of each place are identified as the most place-informative elements, so to distinguish a selected area from others: landmarks, historical architectures, religious sites etc. along with some unusual natural landscapes. More than 2 million social media photos from 18 cities worldwide have been considered, so to simulate a "moving through" perceptive-based experience. Eventually the authors foresee a future research development, to seek connections between visual features and the culture, geography and historical evolution of a city or a landscape.

The same research group with other authors [Zhang, Zhou, Liu et al. 2018] proposes a similar data-driven machine learning approach, better adapted to measure how people perceive a place in a large-scale urban region. Specifically, a deep learning model, which has been trained on millions of human ratings of street-level imagery, was used to predict human perceptions of a street view image. This model can help to map the distribution of the city-wide human perception for a new urban region. From the 150 object categories 
segmented from the street view images (again, according to a 'moving through' approach), various objects were identified as being positively or negatively correlated with six perceptual indicators. The results take researchers and urban planners closer to understanding the interactions of the place sentiments and semantics.

Another group of researchers [Srivastava, Lobry, Tuia et al. 20I 8] suggest using a machine learning approach based on deep learning so to produce land-use maps directly from both the pictures available from Google Street View and OpenStreetMap annotations. Because of the large availability of these two data sources, the proposed approach is scalable to any place and presents the possibility of frequent updates of the resulting map. As base information, the features extracted from single pictures around the object of interest are issued from pre-trained convolutional neural networks. As a result, a machine learning method predicts land-use at the urban-object level, using freely available place based photographies (Google Street View) and crowdsourced land-use information (OpenStreetMap), so to finally produce a map that can integrate at the same time a 'moving through' and a 'looking from above' approach.

A research aiming at labeling urban land use at pixel level, using high-resolution aerial images and ground-level street view images matches both approaches, considering a place at the same time with a 'looking from above' and 'moving through' approach [Cao, Zhu, Tu, Li 2018]. A deep neural network is used to extract semantic features from sparsely distributed street view images and to interpolate them in the spatial domain and to match the spatial resolution of the aerial images, which are finally fused together through a deep neural network for classifying land use categories. The method has been tested on a large publicly available dataset of New York City area. The results show that it is possible to predict urban land use with relatively high accuracy starting from very high-resolution overhead images. At the same time, ground-level street view images contain useful information for land use classification, and the integration of both sources can improve the pixel-level classification results. The impact of aerial imagery resolution also helps in understanding changes on land use classification, and the final results indicate that aerial imagery resolution is positively correlated with classification accuracy, using street view images mainly to enhance the classification accuracy when the resolution of aerial images is lower.

\section{A GIS-based method for measuring spatial quality of slow routes}

In this section a multi indicators GIS-based method has been applied to a slow mobility route (the so called Cammino dei Monaci - The Monk's Route), between Milano and Corte Sant'Andrea (total length $64 \mathrm{~km}$ ), with the aim of measuring the spatial quality of the slow route, by using mapping techniques. The Spatial Quality Index of Slow Routes (SQI$\mathrm{SR}$ ) method is based on a set of heterogeneous indicators (fig. I), that can be measured through quantitative GIS operations, based on the processing of spatial entities (points,

\begin{tabular}{|l|l|l|c|c|}
\hline MACRO CRITERIA & INDICATORS & MEASUREMENT & UNITS & SCORE \\
\hline CULTURAL HERITAGE & Cultural heritage density & Number of cultural heritage & {$[-]$} & $0-1$ \\
\hline \multirow{5}{*}{ LANDSCAPE } & Land use diversity & Number of land uses & {$[-]$} & $0-1$ \\
\cline { 2 - 5 } & Waterways & Waterways length & {$[\mathrm{m}]$} & $0-1$ \\
\cline { 2 - 5 } & Vegetation & Row of trees length & {$[\mathrm{m}]$} & $0-1$ \\
\cline { 2 - 5 } & Protected areas & Parks area percentage & {$[-]$} & $0-1$ \\
\hline $\begin{array}{l}\text { INFRASTRUCTURAL } \\
\text { CONNECTIVITY }\end{array}$ & Road connectivity & Number of roads intersections & {$[-]$} & $0-1$ \\
\cline { 2 - 5 } & Railway and metro connectivity & $\begin{array}{l}\text { Number of railway and metro } \\
\text { stations }\end{array}$ & {$[-]$} & $0-1$ \\
\hline \multirow{3}{*}{ ROUTE-SPECIFIC FEATURES } & Comfort & Paved surface percentage & {$[-]$} & $0-1$ \\
\cline { 2 - 5 } & User-frendliness & Steepness & {$[\mathrm{m}]$} & $0-1$ \\
\cline { 2 - 5 } & Directness & Most direct and current route ratio & {$[-]$} & $0-1$ \\
\hline
\end{tabular}


lines and surfaces) [Scandiffio 2019a]. The SQISR method allows to compute a score for each cell $(500 \mathrm{~m} \times 500 \mathrm{~m})$, that is crossed by the route, in the scale 0 - I0, that corresponds to the sum of the measured values for each indicator per each cell [Scandiffio 2019b].

The SQISR method is able to measure the spatial composition of the landscape crossed by the route, by using a top-down approach, that belongs to the map-making process (looking from above). On the contrary, assuming that human perception is related to a street-level point of view (moving through), the GIS-based approach needs to be integrated with the Machine Learning $(\mathrm{ML})$ system, with the aim of obtaining a more exhaustive method for a deeper investigation of this issue. In the current case study the SQISR method has been used to select the most significant images along the whole route, so that they could be representative of the main spatial features of each cell, with the aim to understand whether ML systems could have eventually lead to similar results. Two different tests have been carried out; in the first step, 16 images along the whole route (Milano - Corte Sant'Andrea $64 \mathrm{~km}$ ) have been selected, by considering points of interest (cultural heritage hotspot, urban settlements and landmarks), but also those open spaces, that seem to reveal a level of uncertainty in the score definition. In the second step, I5 images have been selected by analysing I 5 consecutive route-cells (stretch between Chiaravalle, Civesio andViboldone $6 \mathrm{~km}$ long), with the aim to investigate possible correlations between the SQISR method and the ML system, in a continuous sequence of environments (fig.2). The graph shows the variation of SQISR scores in the stretch between Chiaravalle, Civesio and Viboldone, $6 \mathrm{~km}$ long (fig.3). The best scores correspond to the places, where all indicators are represented by values that are higher than the average, and the spatial composition of the landscape reveals the presence of many components (e.g. both in the Chiaravalle and Viboldone cells there are: cultural heritage density, land use diversity, rows of trees, waterways, protected area). On the other hand the worst scores correspond to the places with uniform/poor spatial composition (e.g. Civesio industrial area), where no considerable landscape components concur to make a pleasant image.

Fig. 2. The map shows the SQISR method applied to the graded GPS track of the Monk's Route. that highlights the score distribution along the route and the localization of the selected images for the analysis with $\mathbb{R}$ system. The extract of the map shows the highlighted the cells with their SOISR scores, corresponding to the selected images for the IR analysis in the stretch between Chiara stretch iboto. Viboldone.

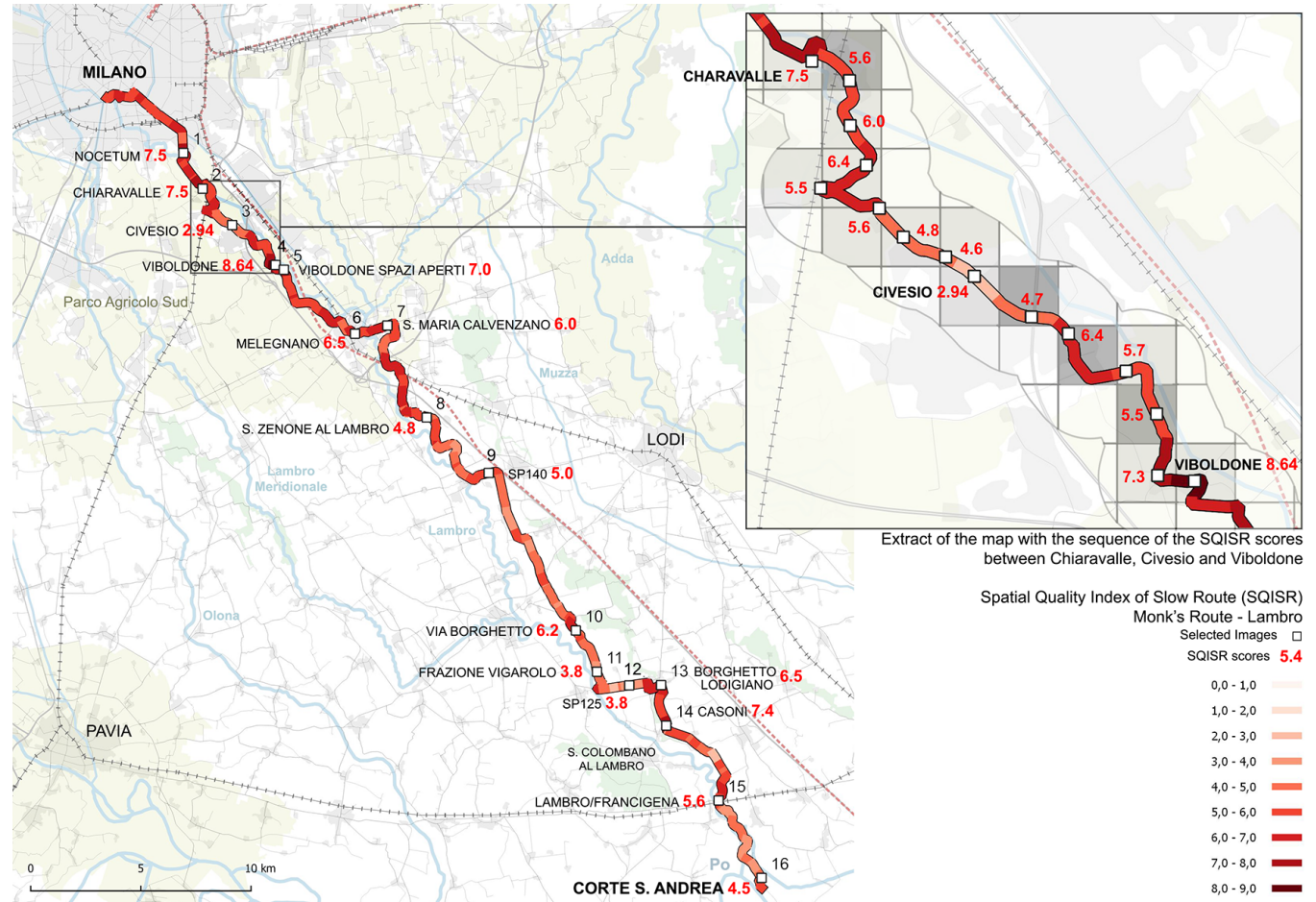


Fig. 3. Graph of the SQISR scores, applied to the stretch between

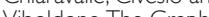
shows the variation shows the variation of the spatial quality cell by cell, and the composition of each score, in relation to the indicators distribution.

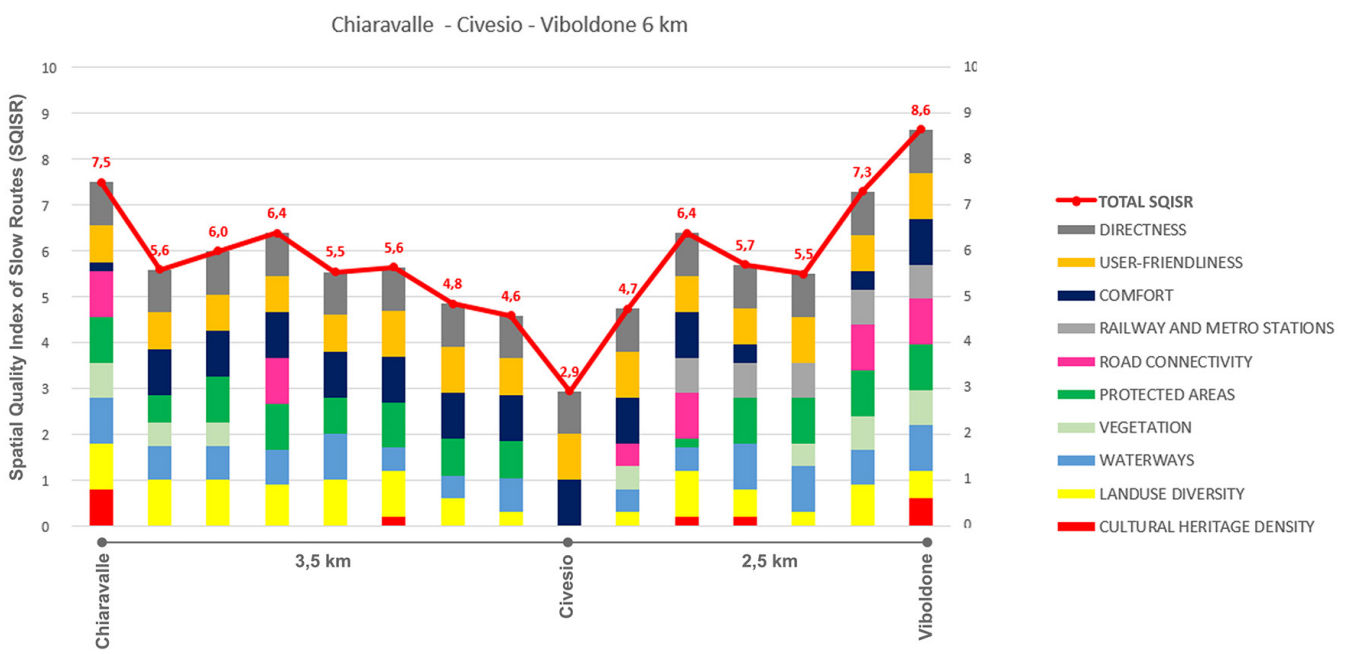

Using Machine Learning technology for measuring the spatial quality

To integrate the SQISR method, an additional cognitive method has been added. The experimentation is still in progress, but first tentative results are worth to be shared. This method is based on the recognition of the elements present on the territory starting from a photographic database widely distributed as Google Street View (GSV), that nowadays represent a big source of information, able to describe whatever place in the world.

The distinction of this work from similar researches - which identify and select the features of urban environment [Zhang, Zhou, Ratti 2019] and [Srivastava, Lobry, Tuia, Vargas-Muñoz 2018]) - is its focus on the outdoors. The images produced by the GSV database are imported into an ML system that recognizes which elements are present in each frame. These must have already been previously acquired by the system through a training procedure that is carried out with a peculiar Machine Learning (ML) process. This procedure carries out a software training through the acquisition of a large number of images in which a known element is present. For each known element, it is associated a label that will be later recognized in an image chosen by the operator.

The most sophisticated ML engines are equipped with automated procedures that detect text, faces, dominant colours, landmarks and labels (tags) of the objects portrayed in the figure. This last procedure is the one used for the recognition of the elements useful for the study and evaluation of the spatial components. When an image taken from the GSV archive has been inserted, the system proceeds to list the percentage probability that an element present in the $M L$ engine database coincides with an element present in the inserted photo. When the recognition procedure has been completed the software produces a list of words and the percentage probability of coincidence. The next step was to aggregate the words into five main semantic fields: road, urban, outdoor, vehicle, heritage within which the tags produced by the ML software engine are included. 16 images extracted from the GSV were sampled and selected in significant locations along the route, (fig. 4) according to the scores of the SQISR method. These raster files are orientated according to a front view, orthogonal to the route direction. The choice to have neutral orientation images is fundamental for the system to work automatically. For each image the $M L$ engine produces a certain number of tags, which are inserted in the respective semantic fields. Finally, the occurrences of each single category are counted and inserted in a histogram that highlights the peculiarities of each shooting area. Three different $M L$ engines were tested, respectively Imagga, Amazon Rekognition and AutoML Vision of Google Cloud Vision with different results in terms of precision, but homogeneous as regards the method of producing the results. The three ML engines have been used in test 
mode. Further research development is currently exploring how to automate the whole process, by writing a proper code in Python to manage API (Application programming interface). At this stage of the research, the $M L$ engines have provided a large set of tags, with large margins of overlap between the used software. After several experimentations of the three ML engines on the two samples of analyzed territory, (figs. 4, 5), some initial considerations can be drawn; final results have been acquired from Google Autovision because of a higher level of accuracy.

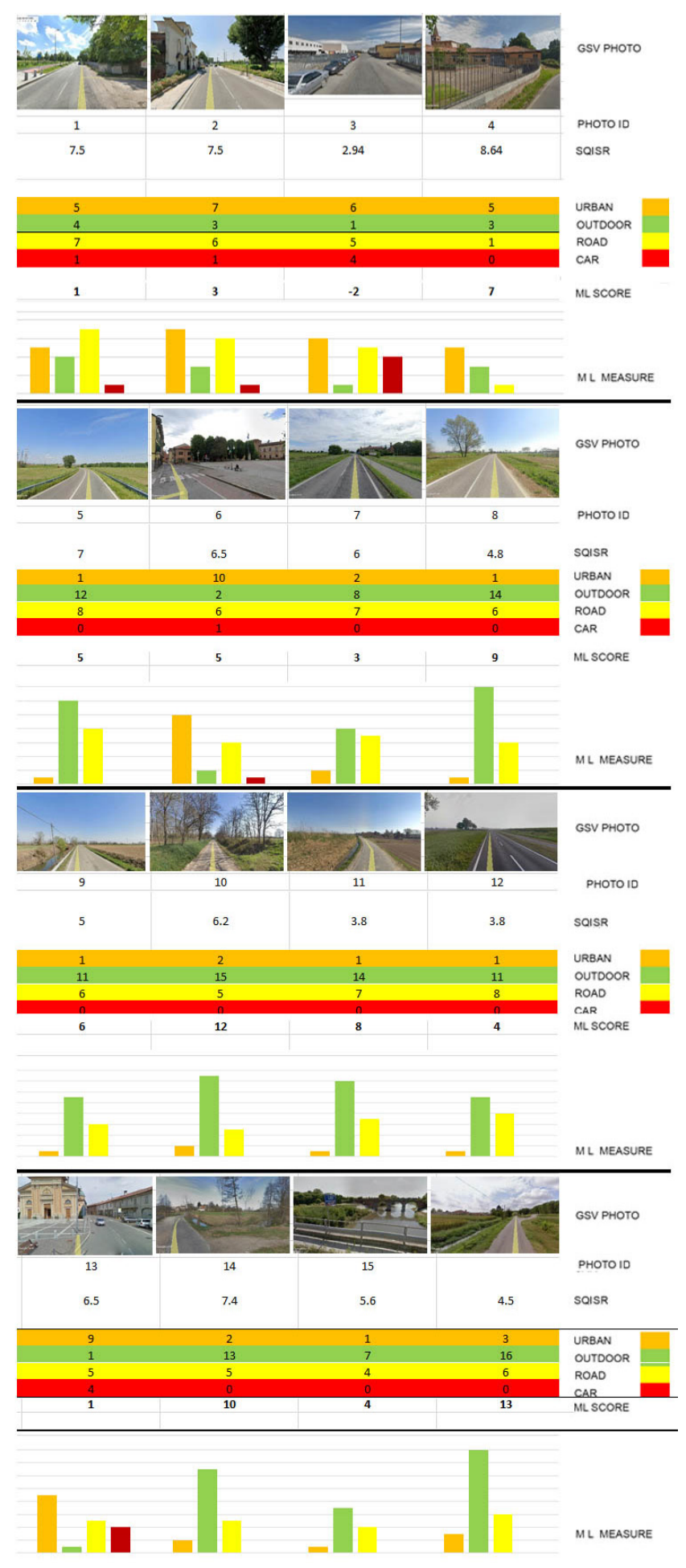


The first outcome is the constant presence of the tag 'road', intuitively present in each sample; therefore this word is considered as a background noise not significant to characterize the route. The second consideration is the difficulty of the system to recognize the words belonging to the semantic field defined as heritage. This is implicitly connected with the image procurement methodology. Using only frontal images with respect to the direction of the route, it is difficult for the ML system to perceive elements of architectural heritage, which only become evident by rotating the point of view. A partial experimentation in this sense has been made, inserting in the $M L$ engine a set of spherical images, with a wider field of view, but the deformation induced by the type of photo has made it difficult to recognize, providing a very limited accuracy. A further experimentation was carried out by selecting three images from the same point of view, the first one frontal and the two lateral ones with 90 degrees rotation around the vertical axis, but the recognition did not lead to optimal results.

A next development of the methodology, will use a range of several images from the same point of view, optimizing the rotation angle of the GSV camera around a vertical axis, leaving the rotation around a horizontal axis unchanged, so to avoid the inclusion of a portion of sky that is not significant for recognition purposes.

The procedure proved to be efficient for the recognition of green elements, both for artificial and natural ones, as the shots taken in non-urbanized areas showed a clear prevalence of tags related to the outdoor semantic field. Intuitively, urban centres where clearly recognized, because a majority of urban elements were detected. For industrial areas, such as Civesio, on the other hand, an important share of road and vehicle elements can be found, which characterize the low spatial quality of the route.

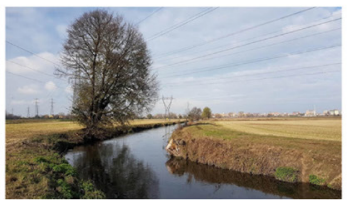

SQISR $=5.5$

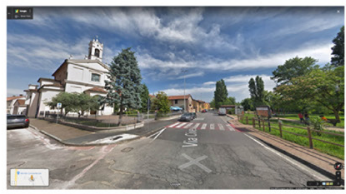

SQISR $=6.4$
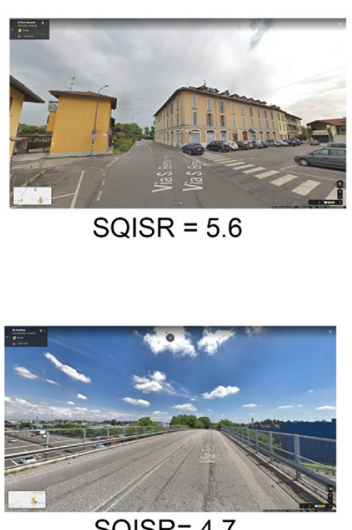

SQISR $=4.7$

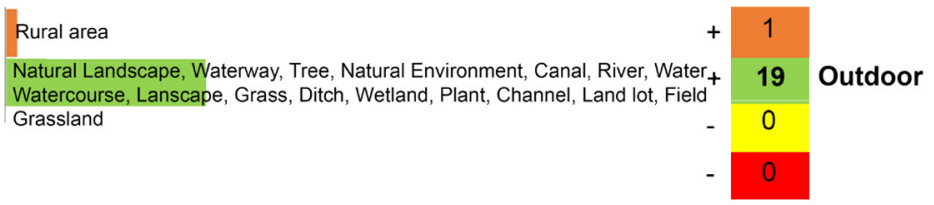

murban moutdoor road @car

ML SCORE $=20$
Fig. 5. Comparison table between Image Recognition and SQISR method. Monk's Route between Chiaravalle, Civesio and Viboldone. Images selected along a route selection of $6 \mathrm{~km}$.

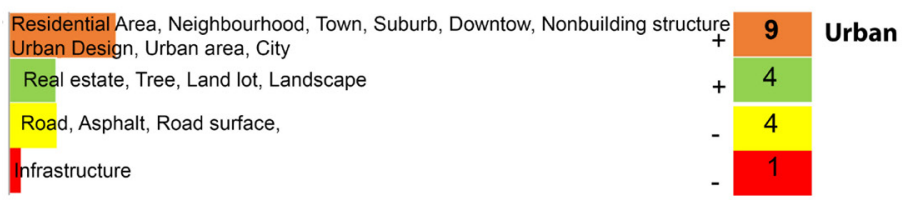

$\mathrm{ML} \mathrm{SCORE}=8$

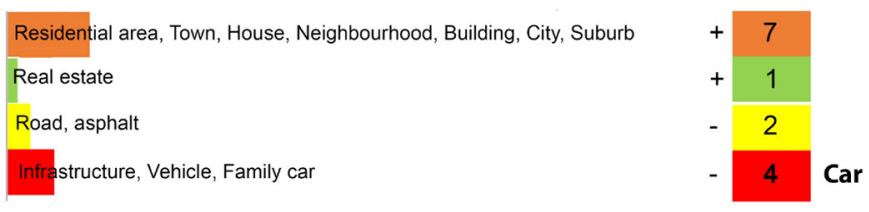

$M L ~ S C O R E=2$

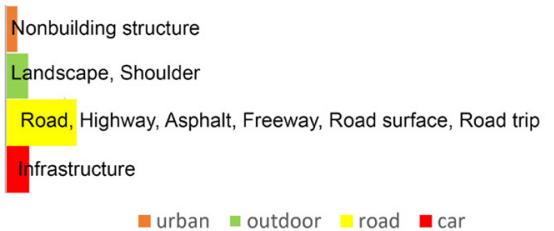




\section{Conclusions}

This paper has shown a work-step of an ongoing research that aims at integrating GIS and ML based methods for measuring spatial quality of slow routes. Many aspects of the research are still to acquire better definition. So far, the applied methodologies give heterogeneous results, especially when valuable elements (mainly from the heritage) are not as well directly visible in photos taken along a defined route that is crossing the same territory. At the same time, using ML tools, when the same spatial elements cannot be detected with the expected level of precision, results can finally be meaningless. From the conducted experimentations, results seem to be promising and both approaches correlate well mainly in areas where natural elements are present. The GIS analysis therefore seems to be indispensable to detect all the landscape elements that are not visible from the GSV images, thanks to the top-down approach of the GIS method.

Another problem that should be solved is the detection of elements belonging to the cultural heritage, that often don't show a clear visual character. In these cases, a further integration with semantic analysis, for instance taken from social networks, should be applied. Further evolution of this research will be based on Machine Learning Image Library customization and improved code-based data acquiring methodology, that could ensure a better integration between the two systems. A step forward will be included with the application of semantic segmentation technology to spatially quantify the presence of single elements in the whole image.

\section{Acknowledgments}

This work has been carried on within the activities of the E-scapes research group (Slow-Foot research grant of CaRiPlo Foundation www.e-scapes.polimi.it directed by A. Rolando) and the Territorial Fragilities Program www.eccellenza.dastu.polimi. it/ (MapFrag group directed by A. Rolando and A. Bianchi) of the Department of Architecture and Urban Studies.

\section{References}

Fan Zhang, Bolei Zhou, Liu Liu, et al. (20 I 8). Measuring human perceptions of a large-scale urban region using machine learning. In Landscape and Urban Planning, I80, p.p. $148-160$.

Fan Zhang, Bolei Zhou, Carlo Ratti (2019). Discovering place-informative scenes and objects using social media photos. In Royal Society open science, 6 , issue 3.

Rolando Andrea, Scandiffio Alessandro (20 I 8). Nodi di reti tangibili e intangibili nel paesaggio tra Torino e Milano. Una sezione territoriale tra i siti Unesco dei Sacri Monti Varallo e di Crea. In Salerno Rossella (a cura di). Rappresentazione Materiale e Immateriale. Atti del $38^{\circ}$ Congresso dei Docenti delle Discipline della Rappresentazione. Milano, I3-I5 Settembre 20 I 8 , pp. | 367- | 372. Roma: Gangemi Editore.

Rui Cao, Jiasong Zhu,Wei Tu, Qingquan Li (20 I 8). Integrating Aerial and StreetView Images for Urban Land Use Classification. In Remote Sensing, 10.

Scandiffio Alessandro (2019a). Spatial quality index of slow routes. A multi-indicators GIS method for measuring spatial quality in the landscape of southern Milan. In Gambardella Carmine. World Heritage and Legacy. Culture, Creativity, Contamination, pp. 505-5 I3. Roma: Gangemi Editore.

Scandiffio Alessandro (2019b). Mapping spatial quality of slow routes with a GIS-based method. A comparative assessment of alternative routes. In International Archives Photogrammetry Remote Sensing Spatial Information Science, XLII-2/W I 5, pp: I07 I-I 076.

Shivangi Srivastava, Sylvain Lobry, Devis Tuia, John E.Vargas-Muñoz (20 I8). Land-use characterisation using Google Street View pictures and OpenStreetMap. In Conference Paper: 2 I th AGILE International Conference on Geographic Information Science, At Lund, Sweden.

\section{Authors}

Alessandro Bianchi, Politecnico di Milano, alessandro.bianchi@polimi.it

Domenico D'Uva, Politecnico di Milano, domenico.duva@polimi.it

Andrea Rolando, Politecnico di Milano, andrea.rolando@polimi.it

Alessandro Scandiffio, Politecnico di Milano, alessandro.scandiffio@polimi.it

To cite this chapter. Bianchi Alessandro, D'Uva Domenico, Rolando Andrea, Scandiffio Alessandro (2020). A View from the Track: measuring spatial quality of slow mobility routes. Possible integration of GIS and machine learning based methods. In Arena A., Arena M., Brandolino R.G., Colistra D., Ginex G., Mediati D., Nucifora S., Raffa P. (a cura di). Connettere. Un disegno per annodare e tessere. Atti del $42^{\circ}$ Convegno Internazionale dei Docenti delle Discipline della Rappresentazione/Connecting. Drawing for weaving relationships. Proceedings of the 42th International Conference of Representation Disciplines Teachers. Milano: FrancoAngeli, pp. 2973-2980. 